University of Nebraska - Lincoln

DigitalCommons@University of Nebraska - Lincoln

$10-18-2006$

\title{
Observation of relativistic cross-phase modulation in high- intensity laser-plasma interactions
}

\author{
Shouyuan Chen \\ University of Nebraska - Lincoln, schen6@unl.edu \\ Matthew Rever \\ University of Nebraska - Lincoln, mrever2@unlserve.unl.edu \\ P. Zhang \\ University of Illinois, Chicago, Illinois \\ W. Theobald \\ University of Rochester, Rochester, New York \\ Donald Umstadter \\ University of Nebraska-Lincoln, donald.umstadter@unl.edu
}

Follow this and additional works at: https://digitalcommons.unl.edu/physicsumstadter

Part of the Physics Commons

Chen, Shouyuan; Rever, Matthew; Zhang, P.; Theobald, W.; and Umstadter, Donald, "Observation of relativistic cross-phase modulation in high-intensity laser-plasma interactions" (2006). Donald Umstadter Publications. 69.

https://digitalcommons.unl.edu/physicsumstadter/69

This Article is brought to you for free and open access by the Research Papers in Physics and Astronomy at DigitalCommons@University of Nebraska - Lincoln. It has been accepted for inclusion in Donald Umstadter Publications by an authorized administrator of DigitalCommons@University of Nebraska - Lincoln. 


\title{
Observation of relativistic cross-phase modulation in high-intensity laser-plasma interactions
}

\author{
S. Chen, ${ }^{1, *}$ M. Rever, ${ }^{1}$ P. Zhang, ${ }^{2}$ W. Theobald ${ }^{3}$ and D. Umstadter ${ }^{1}$ \\ ${ }^{1}$ Department of Physics and Astronomy, University of Nebraska, Lincoln, Nebraska 68588, USA \\ ${ }^{2}$ Physics Department, University of Illinois, Chicago, Illinois 60607, USA \\ ${ }^{3}$ Laboratory for Laser Energetics, University of Rochester, Rochester, New York 14623, USA
}

(Received 14 October 2004; published 18 October 2006)

\begin{abstract}
A nonlinear optical phenomenon, relativistic cross-phase modulation, is reported. A relativistically intense light beam $\left(I=1.3 \times 10^{18} \mathrm{~W} \mathrm{~cm}^{-2}, \lambda=1.05 \mu \mathrm{m}\right)$ is experimentally observed to cause phase modulation of a lower intensity, copropagating light beam in a plasma. The latter beam is generated when the former undergoes the stimulated Raman forward scattering instability. The bandwidth of the Raman satellite is found to be broadened from 3.8-100 nm when the pump laser power is increased from 0.45-2.4 TW. A signature of relativistic cross-phase modulation, namely, asymmetric spectral broadening of the Raman signal, is observed at a pump power of $2.4 \mathrm{TW}$. The experimental cross-phase modulated spectra compared well with theoretical calculations. Applications to generation of high-power single-cycle pulses are also discussed.
\end{abstract}

DOI: 10.1103/PhysRevE.74.046406

PACS number(s): 52.38.-r, 42.65.Jx, 42.65.Re

\section{INTRODUCTION}

Nonlinear optics has been an active research area for decades, ever since lasers reached power levels sufficient to drive atomic electrons anharmonically, and thus modulate the refractive index of an optical medium [1]. With the more recent development of chirped pulse amplification (CPA) [2], peak laser power can now reach the hundred-terawatt level, which, when focused to spot sizes of a few $\mu \mathrm{m}$, can produce peak intensities that can easily exceed $1 \times 10^{18} \mathrm{~W} \mathrm{~cm}^{-2}$. Such high laser intensities drive electrons in plasmas relativistically, providing a different mechanism to modulate the refractive index, and forming the basis for a new regime of nonlinear optics [3]. For example, in recent years, relativistic self-phase modulation (RSPM), relativistic self-focusing, and relativistic harmonic generation have all been observed [4-6], which are analogous to the similar effects found with conventional nonlinear optics. However, there has been no report of another nonlinear optical phenomena with an analog in laser-atom interactions [7], namely, relativistic crossphase modulation (RXPM), which refers to the phase modulation of an optical field induced by another copropagating beam of a different wavelength or polarization. In this paper, we present experimental results demonstrating RXPM of one light pulse (generated by forward Raman scattering) by a separate, relativistically intense, light pulse in a plasma. Besides being of fundamental physical interest, the observation of this novel mechanism may also help to elucidate some previously unexplained results, and may provide a means to generate powerful single-cycle light pulses.

\section{EXPERIMENTAL SETUP}

The experiment was carried out with a 400 fs (FWHM), P-polarized, Ti-sapphire/Nd-glass hybrid laser system with a $1.053 \mu \mathrm{m}$ central wavelength at the University of Michigan FOCUS center. The 2 -in. laser beam was focused by an $f / 3$

\footnotetext{
${ }^{*}$ Corresponding author. Electronic address: schen6@unl.edu
}

parabola onto the edge of a helium gas target generated by a $1.2 \mathrm{~mm}$ diameter supersonic nozzle. The focused laser spot diameter was $12 \mu \mathrm{m}$ FWHM, which contained $60 \%$ of the laser energy. In the experiment, the laser power was varied from 0.45 to $2.4 \mathrm{TW}$. Accordingly, the peak intensity was varied from $2.4 \times 10^{17}$ to $1.3 \times 10^{18} \mathrm{~W} \mathrm{~cm}^{-2}$. The experimental setup is illustrated in Fig. 1. A 2-in. diameter lens with a $30 \mathrm{~cm}$ focal length was used to collimate the light propagating in the forward direction (collection solid angle $\Omega$ $=1.8 \mathrm{msr}$ ), and then a parabolic mirror focused the collimated light onto a spectrometer, which has a detection range from 650 to $1100 \mathrm{~nm}$, with a resolution of $1.3 \mathrm{~nm}$. To improve the contrast of the intensity of the forward Raman satellite with respect to that of the laser, two high reflectors at laser wavelength $(R>98 \%)$ were used to reflect the laser beam, and various combinations of neutral density filters were put in front of the spectrometer to further reduce the light intensity. The laser-plasma interaction distance was monitored from the top of the gas jet by imaging Thomson scattered light from the channel onto a CCD camera.

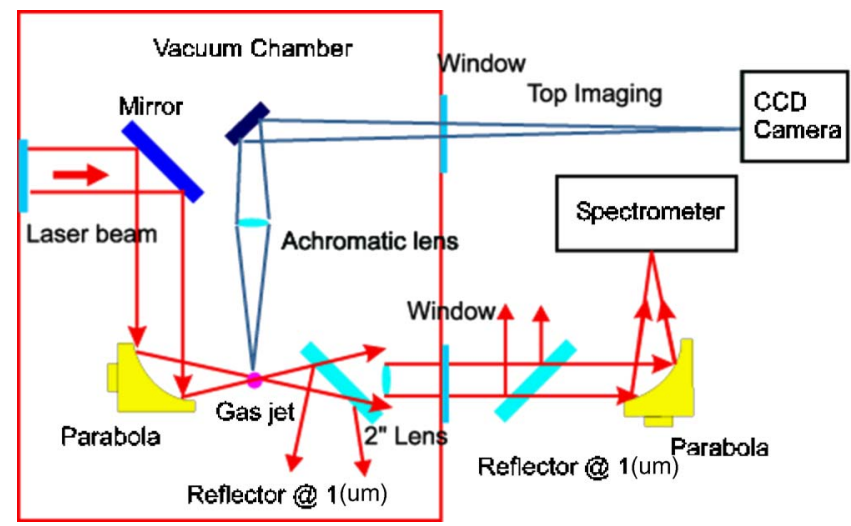

FIG. 1. (Color online) Diagram of the experimental setup. The imaging diagnostic that may appear as side imaging is actually imaging the gas jet from the top. 


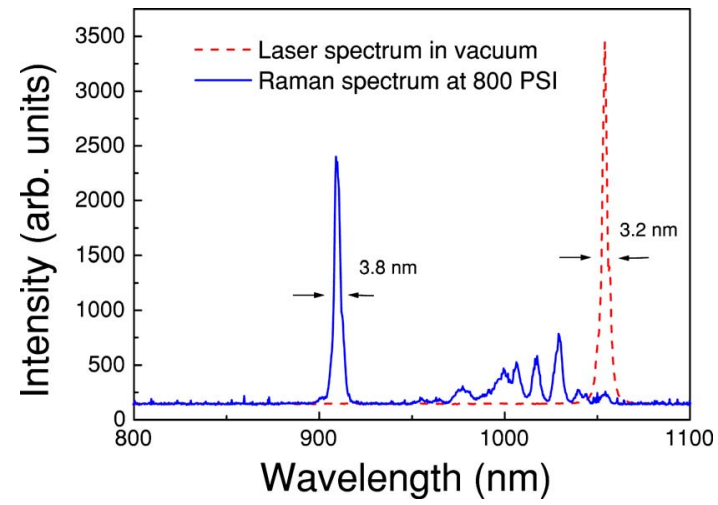

FIG. 2. (Color online) Comparison of the laser spectrum and the Raman spectrum at a helium gas pressure of 800 PSI. The Raman peak wavelength is $905 \mathrm{~nm}$ and the bandwidth is $3.8 \mathrm{~nm}$, which is broader than the laser bandwidth of $3.2 \mathrm{~nm}$. The broadened and blueshifted spectrum near the laser wavelength in the Raman spectrum is due to ionization. The intensity scales of Raman signal and laser are not the same.

\section{EXPERIMENTAL RESULTS AND DATA ANALYSIS}

Figure 2 shows a comparison of the laser pulse spectrum in vacuum and the forward Raman spectrum, which was generated at a laser power of $0.45 \mathrm{TW}$ and a valve backing pressure of 800 PSI. The plasma frequency-measured from the frequency shift of the satellite-was $0.28 \times 10^{15} \mathrm{~s}^{-1}$, and the corresponding plasma density was $2.45 \times 10^{19} \mathrm{~cm}^{-3}$. This was consistent with the gas densities measured by means of interferometry. By moving the gas target position relative to the laser focus position, we found that the Raman signal was strongest when the laser was focused at the edge of the gas target. The intensity of the Raman satellite signal increased with increase of the laser intensity. The bandwidth of the Raman light was not only broadened, but it was also modulated at the laser power higher than 1.2 TW.

As shown in Fig. 3, the Raman signal bandwidth was increased from 4.4 to $5.0 \mathrm{~nm}$ when the laser power was increased from 0.8 to $1.2 \mathrm{TW}$. When the laser power was increased to $2.0 \mathrm{TW}$, a broadened pedestal and modulated side bands began to appear in the Raman spectrum. The pedestal was due to the strongly coupled instability, as was also found in the case of backward Raman scattering [8]. Similarly modulated side bands had been observed in previous experiments without explanation [9]. Since the Raman signal was well defined at a low laser intensity of $2.4 \times 10^{17} \mathrm{~W} \mathrm{~cm}^{-2}$ as shown in Fig. 2, the laser intensity related spectral broadening was not due to the gas target inhomogeneity. Another possible mechanism of spectral broadening is ponderomotive force induced plasma density perturbation, and thus the change of plasma refractive index during the laser-plasma interaction [10]. However, an analysis in the appendix using the parameters of our experiment shows that the ponderomotive force induced phase modulation is about 15 times less than the phase modulation induced by the relativistic effect, and thus it is negligible compared with relativistic phase modulation. For the particular parameters of our experiment, the following analysis shows that this modulation is consistent with RXPM in a plasma.

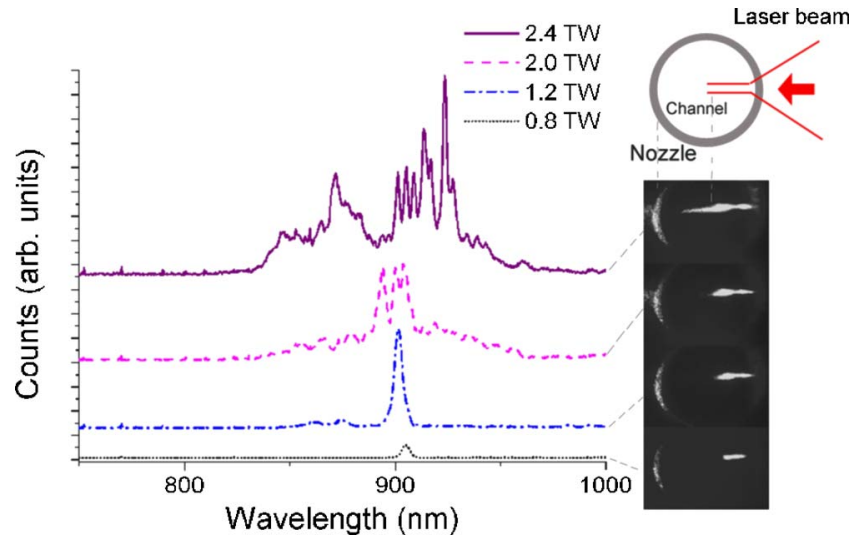

FIG. 3. (Color online) Raman spectra at different laser energies. The inset at right shows topview images, which are related with the corresponding spectra by dashed lines. The schematic of the topview image is shown on the top of the inset. Self-focusing begins to happen at a laser power of 1.2 TW. These spectra are from single reprehensive pulses and they are the typical spectra in the corresponding power level.

A noteworthy aspect of the modulated Raman spectrum was that it was broadened asymmetrically when the laser power reached $2.4 \mathrm{TW}$. One reason for this asymmetric broadening was due to the group velocity mismatch and delay between the laser and the Raman pulse. Because plasma is an anomalous group-velocity dispersion (GVD) medium, the anti-Stokes Raman signal has higher group velocity than that of the laser pulse. Analysis in the latter part of this letter shows that the Raman signal also has more energy earlier in the pulse. As a result, more of the Raman signal's energy rides on the rising edge of the laser pulse than on the falling edge. Since the rising part of the laser pulse introduces a redshift, the broadened Raman spectrum has higher intensity on the redshifted side than the blueshifted side. Because RSPM produces a symmetrically broadened spectrum, this asymmetrical broadening indicates that the broadening of the Raman spectrum is due to RXPM.

Similar to the nonlinear phenomena in gases, liquids, or solids, RXPM in plasmas originates from the intensitydependent refractive index, which, in the case of plasma, is given by $n=\sqrt{1-\omega_{p}^{2} / \omega_{0}^{2}}$, where $\omega_{p}$ is the plasma frequency and $\omega_{0}$ is the central laser frequency. The intensity dependent term is embedded in the expression of the plasma frequency, which is given by $\omega_{p}=\sqrt{4 \pi n_{e} e^{2} / m_{e} \gamma}$, where $n_{e}$ is the plasma density, and $\gamma$ is the relativistic factor of the electrons, which depends on the intensity of the laser field. For a linearly polarized laser field, $\gamma$ is equal to $\sqrt{1+a^{2} / 2}$, where $a$ $=e E / m_{e} \omega c=8.5 \times 10^{-10} \lambda[\mu \mathrm{m}] I^{1 / 2}\left[\mathrm{~W} \mathrm{~cm}^{-2}\right]$ is the normalized vector potential of the laser field. In our experiment, the plasma density is about $0.02 n_{c}$ and the normalized vector potential of the laser field is varied from 0.4 to 1 . Under these conditions, the plasma refractive index can be Taylor expanded, and written as $n=1-\omega_{p}^{2} / 2 \omega_{0}^{2}=1-n_{e} / 2 n_{c} \gamma$, where $n_{c}$ is the critical electron density and is equal to $\omega_{0}^{2} m_{e} / 4 \pi e^{2}$. When $a \leq 1$, the plasma refractive index can be further simplified by expanding about $a$, which results in $n=1-(1$ $\left.-a^{2} / 4\right) n_{e} / 2 n_{c}=n_{1}+n_{2} I$, where $n_{1}=1-n_{e} / 2 n_{c}$ and $n_{2}=(8.5$ 
$\left.\times 10^{-10} \lambda[\mu \mathrm{m}]\right)^{2} n_{e} / 8 n_{c}$. This formula predicts phase modulation and self-focusing in the nonlinear medium.

To further simplify the theoretical analysis, we assume that the plasma is a lossless medium and there is no pump depletion. Because the intensity of the Raman light signal is much weaker than that of the laser beam in our experiment, self-phase modulation of the Raman pulse and its crossphase modulation of the laser beam can be neglected. We also neglect the strong coupling, which should only result in the pedestal under the Raman signal that was observed in the experiment. When the laser pulse undergoes self-focusing, the variation of laser spot size along propagation direction can be neglected and the transverse distribution of electric field is independent of propagation distance $z$. The electric fields of the laser pulse and Raman signal have the form [11]

$$
\widetilde{E}\left(\mathbf{r}, \omega-\omega_{0}\right)=F(x, y) \widetilde{A}\left(z, \omega-\omega_{0}\right) \exp \left(i \beta_{0} z\right),
$$

where $F(x, y)$ is the electric field distribution in transverse direction, $\widetilde{A}(z, \omega)$ is the slow varying function of $z$, and $\beta_{0}$ is the wave number. With these assumptions, the propagation of the laser and stimulated Raman signal can be described by the coupled nonlinear Schrödinger equation as in nonlinear fiber optics [11]

$$
\begin{gathered}
\frac{\partial A_{p}}{\partial z}+\frac{i}{2} \beta_{2 p} \frac{\partial^{2} A_{p}}{\partial T^{2}}=i \delta_{p}\left(\left|A_{p}\right|^{2}\right) A_{p}, \\
\frac{\partial A_{s}}{\partial z}-d \frac{\partial A_{s}}{\partial T}+\frac{i}{2} \beta_{2 s} \frac{\partial^{2} A_{s}}{\partial T^{2}}=i \delta_{s}\left(\left|A_{p}\right|^{2}\right) A_{s}+\frac{g_{s}}{2}\left|A_{p}\right|^{2} A_{s},
\end{gathered}
$$

where $T=t-z / v_{g p}, d=\left(v_{g s}-v_{g p}\right) /\left(v_{g s} v_{g p}\right), \quad \delta=n_{2} \omega_{0} /\left(c A_{\text {eff }}\right)$, $\beta_{2}=1 / c\left(2 d n / d \omega+\omega d^{2} n / d \omega^{2}\right)$.

In the equations, the subscripts $p$ and $s$ represent the laser and Raman pulse, respectively. $A$ is the electric field envelope amplitude of the optical pulse. $A_{\text {eff }}$ is the effective focus area, which can be evaluated from the focal spot size of the laser. $v_{g}$ is the group velocity of the optical field. $T$ is the time measured in the moving frame of pump pulse. $g_{s}$ is the Raman gain coefficient. $\delta$ is the nonlinear coefficient and $\delta_{p}=1.58 \times 10^{-8} \mathrm{~m}^{-1} \mathrm{~W}^{-1}, \quad \delta_{s}=9.80 \times 10^{-9} \mathrm{~m}^{-1} \mathrm{~W}^{-1}$ in our experiment. $d$ is the walk-off parameter by which we can define the walk-off distance $L_{W}=T_{0} /|d|$, which determines the importance of the first derivative in Eq. (3). With the experimental parameters $d=1.09 \times 10^{-11} \mathrm{~s} / \mathrm{m}$ and $T_{0}$ $=400 / 1.665=240 \mathrm{fs}$, the walk-off distance $L_{W}$ is equal to $2.4 \mathrm{~cm} . \quad \beta_{2}$ is the dispersion parameter. $\beta_{2 p}=4.6$ $\times 10^{-26} \mathrm{~s}^{2} \mathrm{~m}^{-1}$ and $\beta_{2 s}=7.2 \times 10^{-26} \mathrm{~s}^{2} \mathrm{~m}^{-1}$. The dispersion distance $L_{D}=T_{0}^{2} /\left|\beta_{2}\right|$ is defined in order to determine the importance of the second derivative in Eqs. (2) and (3). The second derivative in the equation can be neglected if the dispersion distance is much larger than the interaction distance. From the dispersion parameter calculated above, the dispersion distances for the laser and Raman are about 126 and $80 \mathrm{~cm}$, respectively. Since the interaction distance $L$ is about $1 \mathrm{~mm}$, we have $L<L_{W} \ll L_{D}$, and the second derivative term in the coupled equation can be neglected. Under these conditions, the solution of the coupled equations can be written as

$$
\begin{gathered}
A_{p}(L, T)=A_{p}(0, T) \exp \left(i \delta_{p} \phi_{p}\right), \\
A_{s}(L, T)=A_{s}(0, T+L d) \exp \left[\left(g_{s} / 2+i \delta_{s}\right) \phi_{s}\right], \\
\phi_{p}=P_{0} \exp \left(-\tau^{2}\right) L, \\
\phi_{s}=P_{0} \frac{\sqrt{\pi}}{2 \tau}[\operatorname{erf}(\tau+\varsigma)-\operatorname{erf}(\tau)] L,
\end{gathered}
$$

where $\tau=t / T_{0}, \varsigma=L d / T_{0}$, and $P_{0}$ is the laser power.

The solutions show that the laser pulse propagates through the plasma without change in its shape but experiences additional chirp. The Raman pulse not only obtains additional chirp, but also changes pulse duration. Several unknown quantities in the laser-plasma interaction were determined by fitting the theoretically predicted spectrum to the measured experiment spectrum. For example, the laser intensity is usually calculated from the laser focal spot size measured in vacuum at low laser power and the laser power measured by a power meter. When self-focusing happens, the focal spot size will be smaller than the one at low laser power and the calculation will underestimate the laser intensity. From the theoretical equation $P_{c}=17\left(\omega_{0} / \omega_{p}\right)^{2} \mathrm{GW}$, the self-focusing critical power $P_{c}$ is $0.85 \mathrm{TW}$ for the plasma density in our experiment. At a laser power of $2.4 \mathrm{TW}$, the laser intensity in the analysis is $1.9 \times 10^{18} \mathrm{~W} \mathrm{~cm}^{-2}$ instead of $1.3 \times 10^{18} \mathrm{~W} \mathrm{~cm}^{-2}$ from the calculation because of selffocusing. The laser pulse duration is another parameter obtainable from RXPM analysis. The 400 fs laser pulse duration for the laser is measured at the $\mathrm{mJ}$ level. After it passes through the Nd-glass amplifier, the pulse duration will be longer due to the gain narrowing. The measured laser bandwidth in Fig. 2 is $3.2 \mathrm{~nm}$, corresponding to a $510 \mathrm{fs}$, Fouriertransform limited Gaussian pulse. Because of the mismatch between the stretcher and compressor, the laser pulse duration is usually longer than that of Fourier-transform limited pulse. The analysis shows $700 \mathrm{fs}$ fits best the bandwidth of modulated peaks in the measured spectra. The analysis also reveals that the Raman pulse has a pulse duration of $630 \mathrm{fs}$ and has more energy earlier in time. Using other parameters of our experiment, the Raman spectral intensity $\left|A_{s}(L, T)\right|^{2}$ can be plotted. Comparisons of the calculated spectra and experimental spectra at 2.0 and 2.4 TW are shown in Fig. 4. The figure shows good agreement between the theoretical predictions and experimental observations with the exception of the absence of the pedestal in the former case, due to the neglect of strong coupling.

\section{DISCUSSION}

Although RXPM was observed in our experiment through the modulation of a Raman satellite by the laser beam, RXPM can happen whenever a weak probe beam copropagates with a relativistic intensity pump beam. Since the XPM will add extra chirp to the pulse, it can be exploited to compress the probe pulse by passing the chirped pulse through a grating compressor [12]. There is no damage threshold in plasma as with other materials, so plasma can easily support 
a
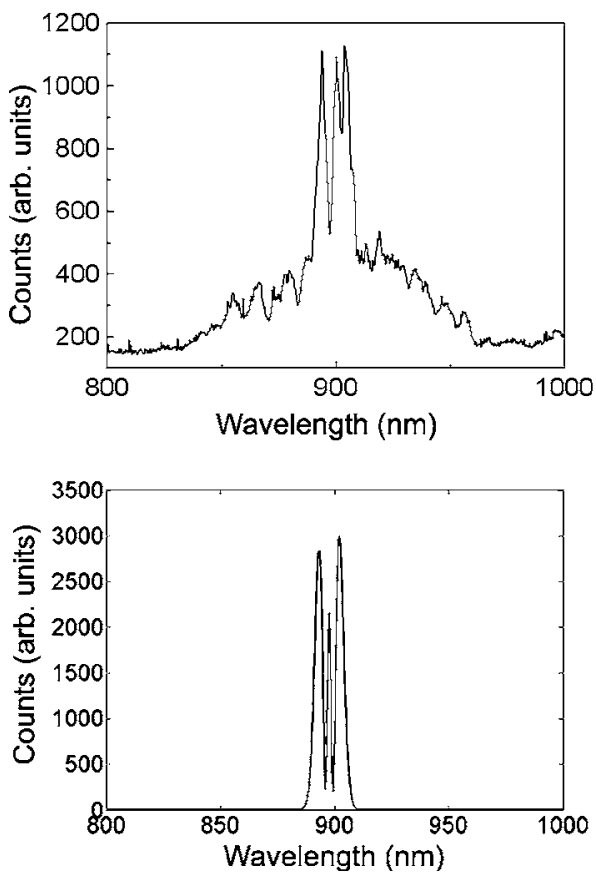

a high-intensity laser pulse. A linear chirp on a probe pulse can be achieved by positioning a short probe pulse at the central part of a long pump pulse since the chirp induced by the phase modulation is approximately linear in the center part of the Gaussian pulse [12]. Spectral adjustment is also helpful for achieving the best chirp for the pulse compression using RXPM. By back propagating a short pulse through the compression system, the required chirp on the probe pulse can be found. The pump and probe pulse parameters will then be adjusted to generate this required chirp. This was proposed by Spanner et al. to generate laser pulses with one or two oscillation cycles [13]. A high power as light pulse can then be produced by high-order harmonic generation [14].

In addition to pulse compression, RXPM can be used as a diagnostic tool to determine the unknown parameters in the laser-plasma interaction as shown in the analysis. Moreover, the plasma wave will also modulate the probe beam and generate a Raman sideband, from which the plasma density can be inferred [15]. The dependence of RXPM-induced chirp on the initial time delay of pump and probe pules can be used to determine the exact delay time between the two pulses.

If the laser intensity is sufficiently high, RXPM needs to be considered even for counterpropagation [16], especially for Raman backscattering from the interaction of high-power laser pulse with plasma [17]. As the backscattered Raman signal is generated with the laser pulse and grows as it propagates back, it will pass the rising and falling part of the laser pulse, so it will be cross-phase modulated. This might explain the broadened backscattered Raman spectrum observed by Rousseaux et al. in their high-power laser-plasma interaction experiment [18]. RXPM also needs to be considered for Raman backscattering amplification when the laser intensity reaches to relativistic regime [19]. Since the plasma is an anomalous GVD medium, the chirped Raman pulse will be
FIG. 4. Comparison of experimental data (top) and analysis results (bottom) shows good agreement. (a) The RXPM Raman spectrum at $2.0 \mathrm{TW}$. The propagation distance is $400 \mu \mathrm{m}$ as measured from the top view image. (b) The RXPM Raman spectrum at $2.4 \mathrm{TW}$. The laser intensity used in the analysis is 1.9 $\times 10^{18} \mathrm{~W} \mathrm{~cm}^{-2}$ instead of 1.3 $\times 10^{18} \mathrm{~W} \mathrm{~cm}^{-2}$ from the calculation. The propagation distance is $1000 \mu \mathrm{m}$. The relatively higher intensity required in the analysis is due to the effect of selffocusing, which increased the laser intensity. The pedestal in the experimental data is due to the strong coupling, which is not included in the model. self-compressed in the plasma [20]. This will affect the predicted gains from the Raman-seeding mechanism [21]. With amplification and compression occurring simultaneously, relativistic plasma is a promising medium for generating high-power, ultrashort laser pulses.

\section{SUMMARY}

In summary, we presented what are- to our knowledgethe first experimental observations of RXPM in a laserplasma interaction. A theoretical model, which is applicable for the analysis of the propagation of a guided laser in a plasma channel, was proposed and was found to compare well with the experimental results. Possible applications of RXPM, such as single-cycle pulse generation and plasma diagnostics, were also discussed.

\section{ACKNOWLEDGMENTS}

This work was supported by the Chemical Sciences, Geosciences, and Biosciences Divisions of the Office of Science, U.S. Department of Energy and the National Science Foundation. Parts of this study were conducted at Focus Center, University of Michigan, Ann Arbor, MI 48109.

\section{APPENDIX: CONSIDERATION ON PONDEROMOTIVE FORCE INDUCED PHASE MODULATION}

Ponderomotive force induced phase-modulation can be understood as follows. When a laser beam is focused into a plasma, a transverse ponderomotive force, which is generated by the gradient of transverse laser intensity, expels electrons out of the laser path and creates plasma density perturbation. Ions are too heavy to respond to the ponderomotive force when the laser pulse duration is less than a picosecond [22]. As the electrons separate from the ions, a static electric 
+ field is generated and eventually balances the ponderomotive force. Since the intensity of a laser pulse usually has a Gaussian distribution in the time domain, the transverse ponderomotive force acting on the electrons also changes with time. This yields a time dependent electron density perturbation, and thus a time dependent plasma refractive index. The time dependent refractive index then modulates the phase of the laser pulse [10]. It is similar to the relativistic phase modulation, in which the time dependent plasma refractive index is induced by the mass change of relativistic electron and correlated with the laser intensity. Since these two effects happen simultaneously in laser-plasma interactions, the question reduces to which mechanism dominates the phase modulation observed in our experiment.

To model the electron density variation induced by the ponderomotive force, we assume that ions form an immobile background and the electron density variation is determined by the balance of the ponderomotive force and electric force that is generated by the charge separation of the electrons and ions. The relative importance of ponderomotive force and relativistic effect can be compared under the same experimental conditions. The laser intensity with a Gaussian spatial profile can be written as

$$
I(r, t)=I_{0}(t) \exp \left(-r^{2} / r_{0}^{2}\right),
$$

the ponderomotive force in transverse direction is

$$
F_{\text {pon }}=m_{e} c^{2} \frac{\partial \gamma}{\partial r}
$$

where $\gamma=\sqrt{1+a^{2} / 2}$ is the relativistic factor of electron, $a$ $=8.5 \times 10^{-10} \lambda_{L}(\mu \mathrm{m}) \sqrt{I\left(\mathrm{~W} / \mathrm{cm}^{2}\right)}$ is the normalized vector potential of the laser field, and $\lambda_{L}$ is the laser wavelength. Using the highest laser intensity in our RXPM experiment, $1.38 \times 10^{18} \mathrm{~W} / \mathrm{cm}^{2}$ and laser wavelength of $1.053 \mu \mathrm{m}$, the normalized vector potential can be expressed as

$$
a=1.05 \exp \left(-r^{2} / 2 r_{0}^{2}\right) \exp \left(-t^{2} / 2 T_{0}^{2}\right),
$$

where $T_{0}=\tau / 1.665, r_{0}=2 R / 1.665, \tau(400 \mathrm{fs})$ is the laser pulse duration (FHWM) and $R(6 \mu \mathrm{m})$ is the measured laser focal radius (FHWM). From Eq. (A2), the ponderomotive force is

$$
F_{\text {pon }}=\frac{m_{e} c^{2} a^{2}}{2 \gamma} \frac{r}{r_{0}^{2}} \text {. }
$$

Since the ponderomotive force is balanced by the electric field generated by the charge separation of the electrons and ions, the electric field can be inferred from the ponderomotive force. The plasma density perturbation can then be calculated from the electric field, which is

$$
E=\frac{F}{e}=\frac{m_{e} c^{2} a^{2}}{2 \gamma e} \frac{r}{r_{0}^{2}}
$$

Since ponderomotive force has a cylindrical symmetry, the electron distribution can be expressed as

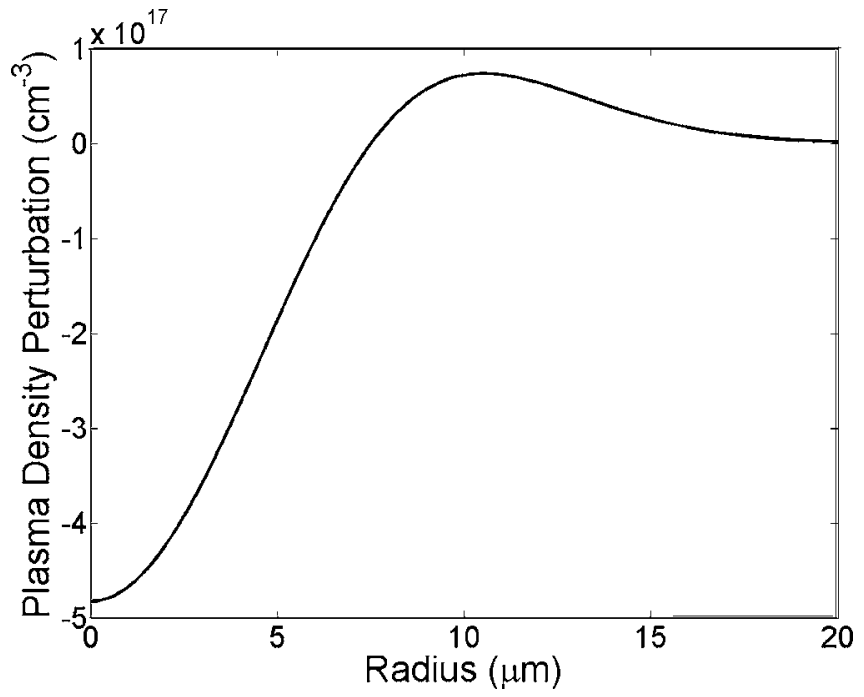

FIG. 5. Plot of electron density perturbation in the radial direction assuming a balance of ponderomotive force generated by the gradient of laser intensity and electric force generated by the charge separation.

$$
\begin{aligned}
\delta n_{e}(r)= & -\frac{\rho(r)}{e}=-\left(\frac{\varepsilon_{0}}{e}\right)\left(\frac{\partial E}{\partial r}+\frac{E}{r}\right) \\
= & -\frac{m_{e} c^{2} \varepsilon_{0}}{e^{2}} \frac{a^{2}}{r_{0}^{2} \sqrt{1+a^{2} / 2}} \\
& \times\left(\frac{r^{2} a^{2}}{4 r_{0}^{2}\left(1+a^{2} / 2\right)}-\frac{r^{2}}{r_{0}^{2}}+1\right) .
\end{aligned}
$$

Figure 5 shows the plasma density modulation caused by ponderomotive force with the above parameters. The maximum density modulation is about $5 \times 10^{17} \mathrm{~cm}^{-3}$, which is approximately $0.0005 n_{c}$, where $n_{c}=1 \times 10^{21} \mathrm{~cm}^{-3}$ is the critical density for a laser wavelength of $1 \mu \mathrm{m}$. The phase modulation induced by self-focusing (filamentation) can then be estimated as [10]

$$
\frac{\Delta \lambda}{\lambda}=-\frac{1}{2} \frac{L}{c} \frac{d\left(n / n_{c}\right)}{d t}
$$

where $L$ is the laser propagation distance, $d\left(n / n_{c}\right)$ is the electron density variation normalized to the critical density, and $d t$ is the laser pulse duration, which is $400 \mathrm{fs}$. For a $1 \mathrm{~mm}$ propagation distance and a density variation of $0.0005 n_{c}$, the calculated spectral broadening contributed by the filamentation is about $2 \mathrm{~nm}$. This is the upper limit for the spectral broadening by filamentation since we use the highest plasma density perturbation. The phase modulation induced by the relativistic effect can also be estimated by Eq. (A7) with $d\left(n / n_{c}\right)$ calculated from the relativistic effect

$$
\eta \simeq 1-\frac{n}{2 \gamma n_{c}}=1-\frac{n}{2 n_{c} \sqrt{1+a^{2} / 2}} \simeq 1-\frac{n}{2 n_{c}}\left(1-\frac{a^{2}}{4}\right) .
$$

With the same experimental conditions, the equivalent plasma density perturbation induced by the relativistic effect 
is $0.28 n / n_{c}$ and $d\left(n / n_{c}\right)$ is 0.0069 . Using Eq. (A7) with the same laser duration and propagation distance, the spectrum broadening induced by relativistic effect is about $30 \mathrm{~nm}$. This is 15 times more than the phase modulation induced by ponderomotive force. This estimation of the spectral broadening applies to both the Raman wave and the fundamental wave, since the Raman and laser wavelengths are close and the refractive index is not much different. Hence the spectral broadening of the Raman wave is also on the order of $30 \mathrm{~nm}$ due to relativistic effects which is about the same as in the measured spectrum.

Though the ponderomotive force can be enhanced by relativistic self-focusing [23], simulation and analysis showed the self-focusing can be strongly reduced by the near Raman forward scattering and Raman side scattering, which scatter the laser energy out of the plasma channel [24]. Selffocusing is further prevented by the heating of the electrons by the forward Raman scattering [25]. Using the similar experimental parameters used in our experiment, the simulation showed that the laser intensity increased 20-80\% depending on the electron temperature [25]. This estimation is consistent with the intensity used in our analysis, which is about $50 \%$ higher than the intensity calculated from the laser focus size in vacuum. At this condition, the relativistic effect still dominates the phase modulation and our model gives good agreement with the experimental observation.
[1] P. A. Franken, A. E. Hill, C. W. Peters, and G. Weinreich, Phys. Rev. Lett. 7, 118 (1961).

[2] D. Strickland and G. Morou, Opt. Commun. 56, 219 (1985).

[3] D. Umstadter and T. Norris, IEEE J. Quantum Electron. 33, 1877 (1997).

[4] C. E. Clayton, K. C. Tzeng, D. Gordon, P. Muggli, W. B. Mori, C. Joshi, V. Malka, Z. Najmudin, A. Modena, and A. E. Dangor, Phys. Rev. Lett. 81, 100 (1998).

[5] I. Watts, M. Zepf, E. L. Clark, M. Tatarakis, K. Krushelnick, A. E. Dangor, R. Allott, R. J. Clarke, D. Neely, and P. A. Norreys, Phys. Rev. E 66, 036409 (2002).

[6] S. Y. Chen, A. Maksimchuk, and D. Umstadter, Nature (London) 396, 653 (1998).

[7] P. D. Maker, R. W. Terhune, and C. M. Savage, Phys. Rev. Lett. 12, 507 (1964).

[8] C. B. Darrow, C. Coverdale, M. D. Perry, W. B. Mori, C. Clayton, K. Marsh, and C. Joshi, Phys. Rev. Lett. 69, 442 (1992).

[9] D. Umstadter, S.-Y. Chen, A. Maksimchuk, G. Mourou, and R. Wagner, Science 273, 472 (1996).

[10] P. E. Young and P. R. Bolton, Phys. Rev. Lett. 77, 4556 (1996).

[11] G. P. Agrawal, Nonlinear Fiber Optics (Academic Press, New York, 1995), Chaps. 2 and 8. Note that, since the nonlinear effect of plasma is from electron relativistic factor $\gamma$ instead of third order susceptibility $\chi^{(3)}$, there is no factor of 2 in front of the XPM term in Eq. (3) as in conventional nonlinear optical equation.

[12] J. T. Manassah, Opt. Lett. 13, 755 (1998).
[13] M. Spanner, M. Ivanov, V. Kalosha, J. Hermann, D. Wiersma, and M. Pshenichnikov, Opt. Lett. 528, 749 (2003).

[14] M. Hentschel, R. Kienberger, C. Spielmann, G. A. Reider, N. Milosevic, T. Brabec, P. Corkum, U. Heinzmann, M. Drescher, and F. Krausz, Nature (London) 414, 509 (2001).

[15] S. P. Le Blanc, M. C. Downer, R. Wagner, S.-Y. Chen, A. Maksimchuk, G. Mourou, and D. Umstadter, Phys. Rev. Lett. 77, 5381 (1996).

[16] B. V. Vu, A. Szoke, and O. L. Landen, Opt. Lett. 18, 723 (1993).

[17] S. C. Wilks, W. L. Kruer, E. A. Williams, P. Amendt, and D. C. Eder, Phys. Plasmas 2, 274 (1995).

[18] C. Rousseaux, G. Malka, J. L. Miquel, F. Amiranoff, S. D. Baton, and P. Mounaix, Phys. Rev. Lett. 74, 4655 (1995).

[19] V. M. Malkin, G. Shvets, and N. J. Fisch, Phys. Rev. Lett. 82, 4448 (1999).

[20] O. Shorokhov, A. Pukhov, and I. Kostyukov, Phys. Rev. Lett. 91, 265002 (2003).

[21] K. Nakajima, D. Fisher, T. Kawakubo, H. Nakanishi, A. Ogata, Y. Kato, Y. Kitagawa, R. Kodama, K. Mima, H. Shiraga, K. Suzuki, K. Yamakawa, T. Zhang, Y. Sakawa, T. Shoji, T. Nishida, N. Yugami, M. Downer, and T. Tajima, Phys. Rev. Lett. 74, 4428 (1995).

[22] T. Kurki-Suonio, P. J. Morrison, and T. Tajima, Phys. Rev. A 40, 3230 (1989).

[23] G.-Z. Sun, E. Ott, Y. C. Lee, and P. Guzdar, Phys. Fluids 30, 526 (1987).

[24] T. M. Antonsen and P. Mora, Phys. Rev. Lett. 69, 2204 (1992).

[25] K.-C. Tzeng and W. B. Mori, Phys. Rev. Lett. 81, 104 (1998). 